\title{
Boundary Conditions for Thermal Lattice Boltzmann Simulations
}

\author{
Annunziata D’Orazio ${ }^{\star 1}$ and Sauro Succi ${ }^{2}$ \\ 1 Dipartimento di Meccanica e Aeronautica, Università di roma "La Sapienza" \\ Via Eudossiana 18, 00184, Roma, Italy \\ dorazio@iac.rm.cnr.it \\ 2 Istituto per le Applicazioni del Calcolo "Mauro Picone", \\ Consiglio Nazionale delle Ricerche \\ Viale del Policlinico 137, 00161, Roma, Italy \\ succi@iac.rm.cnr.it
}

\begin{abstract}
A boundary condition for temperature and heat flux of a thermal lattice Boltzmann method is presented. A thermal lattice BGK model with doubled populations is used to simulate hydrodynamic and thermal fields for flows with viscous heating. The unknown thermal distribution functions at the boundary are assumed to be equilibrium distribution functions with a counter-slip internal energy density which is determined consistently with Dirichlet and/or Neumann boundary conditions.
\end{abstract}

\section{Introduction}

In the last decade lattice kinetic theory, and most notably the Lattice Boltzmann method (see references [1] - [3]), have met with significant success for the numerical simulation of a large variety of athermal fluid flows, including realworld engineering applications. The simulation of flows with significant heat transfer turned out to be much more difficult (see reference [4],[5]). However, in the recent time a number of proposals have appeared (for a review see reference [3], Chapter 14, and reference [6], [7]). Some of these proposals are based on the idea of dispensing with the explicit representation of heat flux in terms of kinetic moments of the particle distribution function $f(\boldsymbol{x}, \boldsymbol{v}, t)$. This idea is interesting because the failure to reproduce the whole set of kinetic moments with only a finite set of discrete speeds, is arguably the major source of numerical instabilities of thermal lattice kinetic equations. In addition, the corresponding lattices need a high number of discrete speeds, which can seriously impair the implementation of boundary conditions, a vital issue for practical applications (see references [8]-[12]). A successfull strategy consists of solving the temperature equation independently of LBE (see reference [6]) and couple to LBE by expressing the fluid pressure as the gradient of an external potential. Clearly,

\footnotetext{
* thanks to "Istituto delle Applicazioni del Calcolo" (IAC-CNR) for kind hospitality during the development of this work.
} 
this strategy represents a drastic departure from a fully kinetic approach, and lacks some elegance. A more elegant possibility is to double the degrees of freedom and express thermal energy density and heat flux as kinetic moments of a separate 'thermal' distribution $g(\boldsymbol{x}, \boldsymbol{v}, t)$. The advantage of this latter approach is that no kinetic moments beyond the first order are ever needed, since heat flux is expressed as the first order vector moment of $g$, the method is able to include viscous heating effects and boundary conditions are easily implemented because both $f$ and $g$ live in the same lattice. The price to pay is doubling of the storage requirements. The method has been successfully demonstrated for the case of Couette flow and Rayleigh-Benard convection (see reference [13]). The aim of this paper is to show that the doubled-LBE, with the new boundary conditions described in detail here, works over a pretty wide range of parameters and it can be extended so as to support velocity-pressure boundary conditions, which are of chief interest to practical engineering purposes.

\section{The Thermal LBE Model}

This paper regards the double-species LBE model proposed in reference [13]. In this model the (thermal) energy is represented by a separate distribution function, $g$, which evolves concurrently with the standard particle distribution function $f$. The distribution function $f$ carries mass and momentum according to the usual expressions:

$$
\rho(\boldsymbol{x}, t)=\int f(\boldsymbol{x}, \boldsymbol{v}, t) d \boldsymbol{v}, \quad \rho(\boldsymbol{x}, t) \boldsymbol{u}(\boldsymbol{x}, t)=\int \boldsymbol{v} f(\boldsymbol{x}, \boldsymbol{v}, t) d \boldsymbol{v}
$$

whereas the distribution function $g$ is in charge of thermal energy density and the corresponding heat flux:

$$
\rho e(\boldsymbol{x}, t)=\int g(\boldsymbol{x}, \boldsymbol{v}, t) d \boldsymbol{v}, \quad \boldsymbol{q}(\boldsymbol{x}, t)=\int \boldsymbol{v}^{\prime} g(\boldsymbol{x}, \boldsymbol{v}, t) d \boldsymbol{v}
$$

where $\boldsymbol{v}^{\prime}=\boldsymbol{v}-\boldsymbol{u}$ is the peculiar speed of molecules relative to the fluid flow and $g(\boldsymbol{x}, \boldsymbol{v}, t)=0.5\left|\boldsymbol{v}^{\prime}\right|^{2} f(\boldsymbol{x}, \boldsymbol{v}, t)$.

The discrete distribution functions are postulated to obey a set of lattice BGK equations of the form:

$$
\begin{gathered}
\tilde{f}_{i}\left(\boldsymbol{x}+\boldsymbol{c}_{i} d t, t+d t\right)-\tilde{f}_{i}(\boldsymbol{x}, t)=-\frac{d t}{\tau_{f}+0.5 d t}\left(\tilde{f}_{i}-f_{i}^{e}\right) \\
\tilde{g}_{i}\left(\boldsymbol{x}+\boldsymbol{c}_{i} d t, t+d t\right)-\tilde{g}_{i}(\boldsymbol{x}, t)=-\frac{d t}{\tau_{g}+0.5 d t}\left(\tilde{g}_{i}-g_{i}^{e}\right)-\frac{d t \tau_{g}}{\tau_{g}+0.5 d t} Z_{i} f_{i} .
\end{gathered}
$$

The new variables $\tilde{f}$ and $\tilde{g}$ are defined as

$$
\tilde{f}_{i}=f_{i}+\frac{0.5 d t}{\tau_{f}}\left(f_{i}-f_{i}^{e}\right), \quad \tilde{g}_{i}=g_{i}+\frac{0.5 d t}{\tau_{g}}\left(g_{i}-g_{i}^{e}\right)+\frac{d t}{2} Z_{i} f_{i}
$$


where, for a nine-directional square lattice with $c^{2}=3 R T$, the equilibrium density distribution are chosen as follows:

$$
\begin{gathered}
f_{i}^{e}=w_{i} \rho\left[1+\frac{3 \boldsymbol{c}_{\boldsymbol{i}} \cdot \boldsymbol{u}}{c^{2}}+\frac{9\left(\boldsymbol{c}_{\boldsymbol{i}} \cdot \boldsymbol{u}\right)^{2}}{2 c^{4}}-\frac{3\left(u^{2}+v^{2}\right)}{2 c^{2}}\right] \\
g_{0}^{e}=-w_{0}\left[\frac{3 \rho e}{2} \frac{\left(u^{2}+v^{2}\right)}{c^{2}}\right] \\
g_{\rightarrow, \uparrow, \leftarrow, \downarrow}^{e}=w_{1} \rho e\left[1.5+\frac{1.5 \boldsymbol{c}_{\boldsymbol{i}} \cdot \boldsymbol{u}}{c^{2}}+\frac{4.5\left(\boldsymbol{c}_{\boldsymbol{i}} \cdot \boldsymbol{u}\right)^{2}}{c^{4}}-\frac{1.5\left(u^{2}+v^{2}\right)}{c^{2}}\right] \\
g_{\nearrow, \backslash, \swarrow, \searrow}^{e}=w_{2} \rho e\left[3+\frac{6 \boldsymbol{c}_{\boldsymbol{i}} \cdot \boldsymbol{u}}{c^{2}}+\frac{4.5\left(\boldsymbol{c}_{\boldsymbol{i}} \cdot \boldsymbol{u}\right)^{2}}{c^{4}}-\frac{1.5\left(u^{2}+v^{2}\right)}{c^{2}}\right] .
\end{gathered}
$$

In the above, the velocity is $\boldsymbol{u}=(u, v)$, the thermal energy density is $\rho e=$ $\rho R T$ (in 2D), the weights of the different populations are

$$
w_{0}=\frac{4}{9}, w_{i}=w_{1}=\frac{1}{9} \quad i=\rightarrow, \uparrow, \leftarrow, \downarrow, w_{i}=w_{2}=\frac{1}{36} \quad i=\nearrow, \nwarrow, \swarrow, \searrow
$$

and the arrows indicate the corresponding discrete speeds.

The term $Z_{i}=\left(\boldsymbol{c}_{i}-\boldsymbol{u}\right) \cdot D_{i} \boldsymbol{u}$ represents the effects of viscous heating and $D_{i}=\partial_{t}+\boldsymbol{c}_{i} \cdot \nabla$ is the material derivative along direction $\boldsymbol{c}_{i}$. More specifically:

$$
Z_{i}=\left[\boldsymbol{c}_{i}-\boldsymbol{u}(\boldsymbol{x}, t)\right] \cdot\left[\boldsymbol{u}\left(\boldsymbol{x}+\boldsymbol{c}_{i} d t, t+d t\right)-\boldsymbol{u}(\boldsymbol{x}, t)\right] / d t .
$$

Finally:

$$
\begin{gathered}
\rho=\sum_{i} \tilde{f}_{i}, \quad \rho e=\sum_{i} \tilde{g}_{i}-\frac{d t}{2} \sum_{i} f_{i} Z_{i} \\
\rho \boldsymbol{u}=\sum_{i} \boldsymbol{c}_{i} \tilde{f}_{i}, \quad \boldsymbol{q}=\left(\sum_{i} \boldsymbol{c}_{i} \tilde{g}_{i}-\rho e \boldsymbol{u}-\frac{d t}{2} \sum_{i} \boldsymbol{c}_{i} f_{i} Z_{i}\right) \frac{\tau_{g}}{\tau_{g}+0.5 d t} .
\end{gathered}
$$

To improve the numerical stability, we use space-time independent average values of $T$ to calculate the local equilibria of $\tilde{f}$ (average thermohydrodynamic consistency): $c_{s}^{2} \propto \bar{T}=$ const.

The kinetic viscosity and the thermal diffusivity are given by $\nu=\tau_{f} R \bar{T}$ and $\chi=2 \tau_{g} R \bar{T}$. Note that by going from $f_{i}$ to $\tilde{f}_{i}, \nu$ has the same form in both Navier-Stokes and energy equations .

\section{Boundary Conditions}

In previous works, different types of boundary conditions are adopted for the present model. In [13], the bounce-back rule of the nonequilibrium distribution proposed by [15] is applied to the thermal population. In [16], the local thermal equilibrium distribution functions are applied on the wall nodes in the case of 
known temperature, while the energy distribution on the wall are set equal to those of the nearest interior nodes in the case of adiabatic wall.

As proposed in [14] for the velocity boundary condition at the wall, the "counter-slip" approach as applied to the internal energy, is adopted here. The incoming unknown thermal populations are assumed to be equilibrium distribution functions with a counter-slip thermal energy $e^{\prime}$, which is determined so that suitable constraints are verified, i.e. fixed temperature or heat flux. To explain the procedure, the following refers to a channel where the flow is driven by suitable boundary conditions and forcing terms applied to the populations $\tilde{f}$.

First, the case of a wall with a fixed temperature is discussed. The unknown distribution functions are (North wall, as an example) $\tilde{g}_{\downarrow}, \tilde{g}_{\swarrow}$ and $\tilde{g}_{\searrow}$ and they are chosen as:

$$
\tilde{g}_{i}=\rho\left(e+e^{\prime}\right)[\text { corresponding form for equilibrium }], \quad i=\downarrow, \searrow, \swarrow \quad .
$$

The constraint

$$
\sum_{i} \tilde{g}_{i}=\rho e+\frac{d t}{2} \sum_{i} f_{i} Z_{i}
$$

yields

$$
\rho e^{\prime}=2 \rho e+1.5 d t \sum_{i} f_{i} Z_{i}-3 G .
$$

In the above, $G$ is the sum of the known populations coming from the fluid nodes and the nearest wall nodes, while the component $v$ of the velocity is zero at the wall.

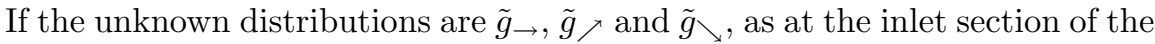
channel, and a temperature profile is imposed, the counter-slip thermal energy is given by:

$$
\rho e^{\prime}=\frac{\rho e\left(4-3 \frac{u}{c}-3 \frac{u^{2}}{c^{2}}\right)+3 d t \sum_{i} f_{i} Z_{i}-6 G}{2+3 \frac{u}{c}+3 \frac{u^{2}}{c^{2}}}
$$

where $G$ is the sum of the known populations coming from the nearest fluid nodes.

In the case of fully developed thermal flow, at the outlet section of a channel, the constraint on the heat flux

$$
\sum_{i} c_{i x} \tilde{g}_{i}=\rho e u+0.5 d t \sum_{i} c_{i x} f_{i} Z_{i}
$$

can be applied and the unknown populations $\tilde{g}_{\leftarrow}, \tilde{g}_{\nwarrow}$ and $\tilde{g}_{\swarrow}$ are determined by the condition:

$$
\rho e^{\prime}=\frac{6\left(\sum_{\rightarrow, \nearrow, \searrow} \tilde{g}_{i}\right)-3 d t \sum_{i} \frac{c_{i x}}{c} Z_{i} f_{i}-\rho e\left(2+3 \frac{u}{c}+3 \frac{u^{2}}{c^{2}}\right)}{2-3 \frac{u}{c}+3 \frac{u^{2}}{c^{2}}} .
$$

If the wall of the channel is adiabatic, the constraint on the heat flux

$$
\sum_{i} c_{i y} \tilde{g}_{i}=0.5 d t \sum_{i} c_{i y} f_{i} Z_{i}
$$


yields (with reference to the South wall where the unknown populations are $\tilde{g}_{\uparrow}$, $\tilde{g}_{\nwarrow}$ and $\left.\tilde{g}_{\nearrow}\right)$

$$
\rho e^{\prime}=3\left(\sum_{\downarrow, \swarrow, \searrow} \tilde{g}_{i}\right)-1.5 d t \sum_{i} \frac{c_{i y}}{c} Z_{i} f_{i}-\rho e .
$$

Finally, the corners can be treated in a similar way and the counter slip procedure can be imposed to the five unknowns incoming at the corner. As an example, at the NW corner the unknown populations are $\tilde{g}_{\leftarrow}, \tilde{g}_{\downarrow}, \tilde{g}_{\searrow}, \tilde{g}_{\nearrow}$ and $\tilde{g}_{\swarrow}$; if the corner node is a fixed temperature wall node, it follows that

$$
\rho e^{\prime}=\frac{\rho e\left(5-4 \frac{u}{c}-5 \frac{u^{2}}{c^{2}}\right)+6 d t \sum_{i} f_{i} Z_{i}-12 G}{7+4 \frac{u}{c}+5 \frac{u^{2}}{c^{2}}} .
$$

\section{Numerical Results}

To demonstrate the accuracy of the method with the present boundary condition, results for Couette and Poiseuille thermal flows are given; for details and more extensive discussion, see reference [17]).

\subsection{Thermal Couette Flow}

A two-dimensional planar flow with a hot wall at temperature $T_{\mathrm{W}}=T_{\mathrm{N}}$, moving along the horizontal direction $x$ at speed $U_{\mathrm{W}}$, is considered. The lower (south) wall is at rest and at constant temperature $T_{\mathrm{S}}$. The walls are at a distance $H$. The major control parameters are the Prandtl number $\operatorname{Pr}=\nu / \chi$ and the Eckert number $E c=U^{2} / c_{v} \Delta T$, constructed with a characteristic velocity. The effects of viscous heating are controlled by the Brinkman number $B r=\operatorname{Pr} E c$, representing the ratio between viscous dissipation and heat condution. The analytical solution for the temperature field is:

$$
T(y)=T_{\mathrm{S}}+\frac{y}{H}\left(T_{\mathrm{N}}-T_{\mathrm{S}}\right)+\frac{B r\left(T_{\mathrm{N}}-T_{\mathrm{S}}\right)}{2} \frac{y}{H}\left(1-\frac{y}{H}\right) .
$$

At inlet and outlet sections periodic boundary conditions for both populations $\tilde{f}$ and $\tilde{g}$ are used. The wall speed $U_{\mathrm{W}}$ has been imposed by means of a pair of self-consistent reflection/slip coefficients $r, s$ as follows:

$$
\begin{gathered}
\tilde{f}_{\uparrow}=\tilde{f}_{\downarrow}, \quad \tilde{f}_{\searrow}=r \tilde{f}_{\swarrow}+s \tilde{f}_{\nearrow}, \quad \tilde{f}_{\swarrow}=r \tilde{f}_{\nearrow}+s \tilde{f}_{\nwarrow} \\
r=1-\frac{\rho U_{\mathrm{W}}}{6 c\left(\tilde{f}_{\nearrow}-\tilde{f}_{\nwarrow}\right)}, \quad s \equiv 1-r .
\end{gathered}
$$

The wall temperature $T_{\mathrm{N}}$ has been imposed by means of the correction (16). The Reynolds number is constructed with $H$ and $U_{\mathrm{W}}$ and the average temperature used in particle equilibria is $\bar{T}=\left(T_{\mathrm{S}}+T_{\mathrm{N}}\right) / 2$. Several tests (not shown here) 
have been performed with $\tau_{f}$ and $\tau_{g}$ ranging from 0.05 to 5.0. Satisfactory results are obtained in all cases. The Figure 1 shows normalized temperature profiles, as compared with analytical solutions, for flows with moderate viscous heating $(B r=0.9)$ and significant viscous heating $(B r=2.93)$. the relative error is $3.4 \times 10^{-4}$ in the first case $(\Delta T=1.0)$ and $1.1 \times 10^{-3}$ in the second $(\Delta T=9.0)$, even with a significant value of $U_{\mathrm{W}}$.

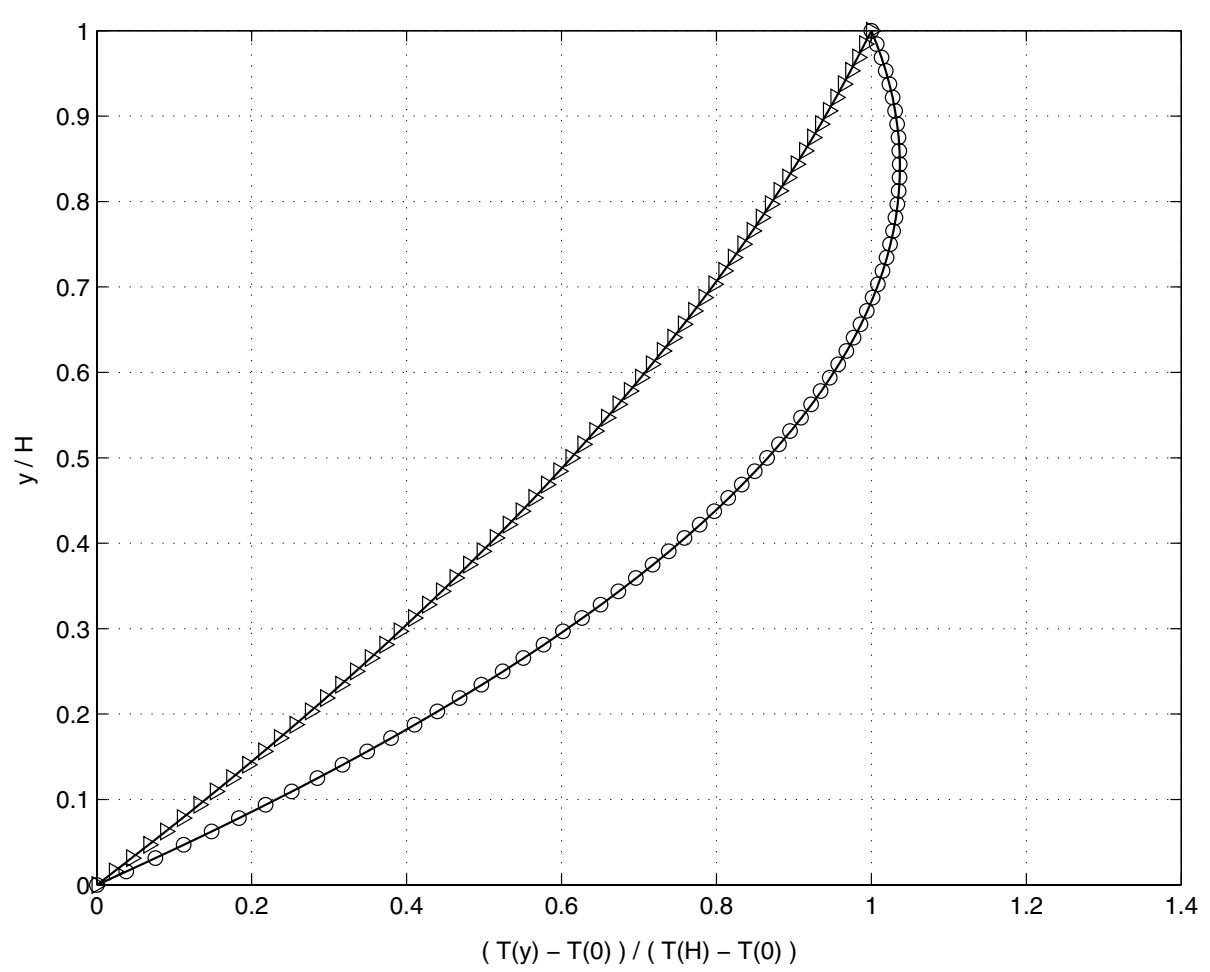

Fig. 1. Couette flow: normalized transverse temperature profiles as compared with analytical results (solid lines). (Triangles): $\tau_{f}=1.0, \tau_{g}=0.1, U_{\mathrm{W}}=0.2, T_{\mathrm{N}}=2.0$, $R e=76.8, E c=0.18, P r=5.0, B r=0.9$ (mild viscous heating). (Circles): $\tau_{f}=5.0$, $\tau_{g}=0.25, U_{\mathrm{W}}=0.4, T_{\mathrm{N}}=10.0, R e=30.72, E c=0.293, \operatorname{Pr}=10.0, \mathrm{Br}=2.93$ (significant viscous heating) 


\subsection{Thermal Poiseuille Flow}

A two dimensional planar flow driven by a constant pressure gradient $d P / d x$, with the walls at rest and at constant temperature $T_{\mathrm{W}}$, is considered. Uniform velocity and temperature profiles $U_{\mathrm{I}}$ and $T_{\mathrm{I}}$, at the inlet section, and thermally fully developed flow, at the outlet section, are imposed. The constant pressure gradient $\Delta P / L$ is simulated by means of a constant volumetric forcing $F_{\text {pois }}=8 \rho_{0} \nu U_{\mathrm{M}} / H^{2}$. The analytical solution for the temperature field (stationary developed solution) is obtained from Navier-Stokes and energy equations for incompressible flows in the limit $R e \rightarrow 0$. It reads as follows:

$$
T(y)=T_{\mathrm{S}}+\frac{\left(T_{\mathrm{N}}-T_{\mathrm{S}}\right) y}{H}+\frac{P r U_{\mathrm{M}}^{2} y}{c_{v} H}\left[\frac{8}{3}-8 \frac{y}{H}+\frac{32}{3}\left(\frac{y^{2}}{H^{2}}\right)-\frac{16}{3}\left(\frac{y^{3}}{H^{3}}\right)\right]
$$

where $U_{\mathrm{M}}$ is the center-line velocity.

The inlet/outlet and wall boundary conditions for the populations $\tilde{f}_{i}$ are imposed by means of the constraints (the example refers to the inlet section):

$$
\sum_{i} \tilde{f}_{i}=\rho, \quad \sum_{i} \tilde{f}_{i} c_{i x}=\rho u+\frac{d t}{2} F_{\text {pois }}, \quad \sum_{i} \tilde{f}_{i} c_{i y}=\rho v=0
$$

plus a "bounce-back" conditions on the non-equilibrium populations (for details we refer the reader to [15] and [17]):

$$
\tilde{f}_{\rightarrow}-f_{\rightarrow}^{e}=\tilde{f}_{\leftarrow}-f_{\leftarrow}^{e}
$$

With respect to the thermal field, the condition (16) is applied to the north wall and a similar constraint holds for the south case; the condition (17) to the inlet section, and the condition (19) to the outlet, are applied. The corners are treated with similar procedure as in (22).

A $1281 \times 65$ grid, corresponding to an aspect ratio $A=20.0$, is used with $\tau_{f}=\tau_{g}=0.5, U_{\mathrm{M}}=0.1, U_{\mathrm{I}}=0.2 / 3.0, T_{\mathrm{I}}=10.0$ and $T_{\mathrm{W}}=1.0$. The Reynolds number constructed with mean velocity and hydraulic diameter is $R e=51.21$ and the average temperature used in particle equilibria is $\bar{T}=\left(T_{\mathrm{I}}+T_{\mathrm{W}}\right) / 2$.

The Figure 2 reports the normalized transverse temperature profile and shows satisfactory agreement for the fluid temperature in the final section of the channel, where the maximum relative error is $1.6 \times 10^{-4}$; at the internal section, the effect of the high inlet temperature is still present.

In Figure 3 are shown results for the normalized transversal heat flux profile at $x / L=0.875$, as compared with analytical results. Again, fairly satisfactory agreement is observed.

Finally, in the case of cooling without viscous heating, the analytical value of the local Nusselt number, corresponding to a hydrodynamically fully developed flow, is $N u_{x}=7.54$. This is defined as $N u_{x}=h_{x} D / k$, where $h_{x}$ is the local convection conductance, $D$ is the hydraulic diameter and $k$ is the thermal conductivity, and is obtained as an infinite-series-solution (see reference [18], 
Chapters 7 and 9 ). In excellent agreement with this value, $N u_{x}=7.56$ is obtained in the thermal fully developed region.

We have to point out that these results are obtained for $\tau_{f}=0.5$. For different values, the temperature profile shows a departure from the analytical solution at the outlet section. We verified that is due to the effect of the non-uniform velocity profile into the equilibria of $\tilde{g}$. For the case of the natural convection in a cavity or the Couette flow described in section 4.1, satisfactory results are obtained for $\tau_{f}$ and $\tau_{g}$ as low as 0.05 .

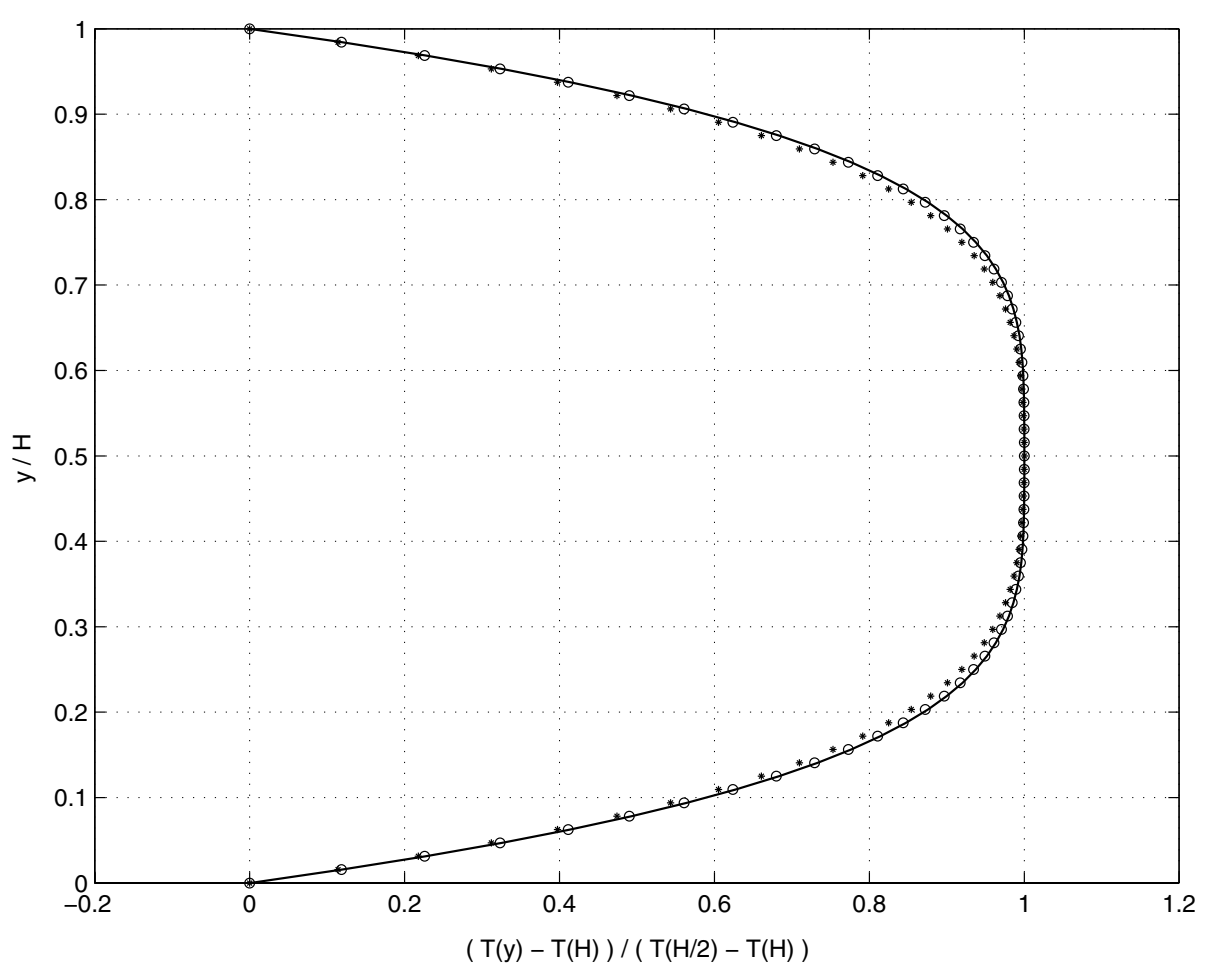

Fig. 2. Poiseuille flow: normalized transverse temperature profile at $x / L=0.75$ (squares) and $x / L=1.0$ (circles); the (solid line) is the analytical solution. $A=20.0$, $\tau_{f}=\tau_{g}=0.5, \operatorname{Pr}=0.5, \operatorname{Re}=51.21, U_{\mathrm{I}}=0.2 / 3, T_{\mathrm{I}}=10.0, T_{\mathrm{W}}=1.0$ 


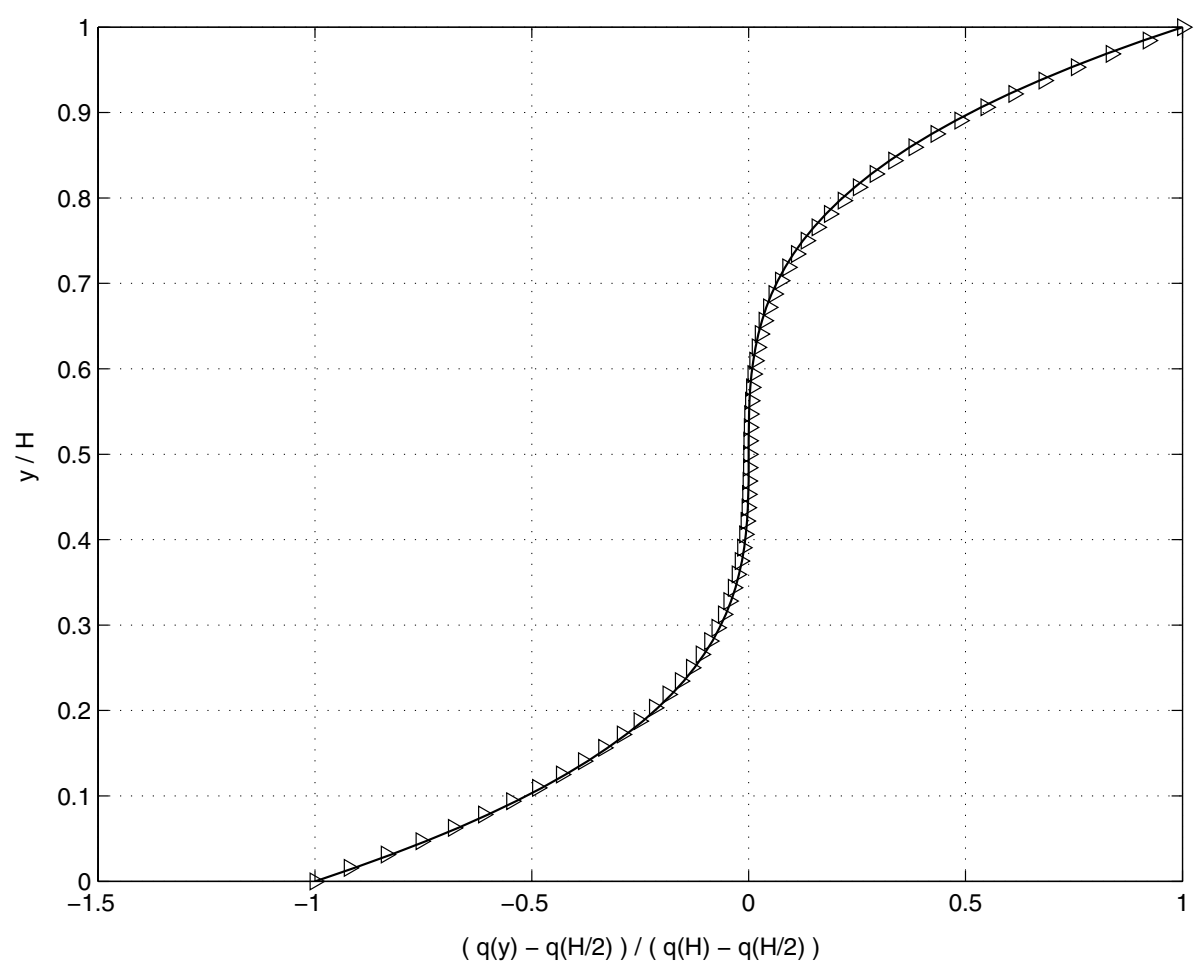

Fig. 3. Poiseuille flow: normalized transverse heat flux profile at $x / L=0.875$; the (solid line) is the analytical solution. $A=20.0, \tau_{f}=\tau_{g}=0.5, \operatorname{Pr}=0.5, \operatorname{Re}=51.21$, $U_{\mathrm{I}}=0.2 / 3, T_{\mathrm{I}}=10.0, T_{\mathrm{W}}=1.0$

\section{Conclusions}

Summarizing, the implementation of a suitable set of boundary conditions for thermal Lattice Boltzmann schemes with doubled populations has been discussed. This study shows that:

1) The doubled thermal scheme provides reliable results over a wide range of physical parameters, including situations with strong temperature contrasts and/or significant viscous heating effects.

2) A new type of boundary condition for the temperature provides good results for both Dirichlet and Neumann conditions.

3) By applying a hybrid set of boundary conditions, in which the flow is forced by a volumetric source of momentum, and still imposing the inlet velocity profile, the method can be adapted to situations of engineering interest.

4) Work is in progress to improve the results for the case of non-uniform velocity profile at the domain boundaries. 


\section{References}

[1] F. Alexander, S. Schen, J. Sterling, "Lattice Boltzmann thermohydrodynamics", Phys. Rev. E, 53, 2298, (1993).

[2] R. Benzi, S. Succi, M. Vergassola, "The lattice Boltzmann equation: theory and applications", Phys. Rep., 222, 145, (1992).

[3] S. Succi, "The Lattice Boltzmann equation", Oxford Univ. Press, Oxford, (2001).

[4] H. Chen, "H-theorem and generalized semi-detailed balance condition for lattice gas system", J. Stat. Phys., 81, (1995).

[5] I. V. Karlin, A. Ferrante, H. C. Öttinger, "Perfect entropy functions of the Lattice Boltzmann method", Europhys. Lett., 47, 182, (1999)

[6] L. S. Luo, "Some recent results on discrete velocity models and ramifications for lattice Boltzmann equation", Proceedings of LGA99, "Discrete simulation of fluid dynamics", Tokyo, July 99, Comp. Phys. Comm. 129, 263, (2000).

[7] P. Lallemand, L. S. Luo, "Hybrid Finite-Difference Thermal Lattice Boltzmann Equation", Proceedings of 11th Conf. on "Discrete Simulation of Fluid Dynamics and Soft Condensed Matter", Shanghai, (2002), to appear in Int. J. Mod. Phys. B.

[8] Y. Chen, H. Ohashi, M.Akiyama, "Thermal lattice Bhatnagar-Gross-Krook model without non-linear deviations in macrodynamic equations", Phys. Rev. E, 50, 2776, (1994).

[9] Y. Chen, H. Ohashi, M.Akiyama, "Prandtl number of lattice Bhatnagar-GrossKrook fluid", Phys. Fluids, 7, 2280, (1995).

[10] G. Mc Namara, A. Garcia, B. Alder, "Stabilization of thermal Lattice Boltzmann models", J. Stat. Phys. 81, 395, (1995).

[11] A. Renda, S. Succi, I. Karlin, G. Bella, "Thermohydrodynamic lattice-BGK equilibria with non-perturbative equilibria", Europhys. Lett. 41, 279, (1998).

[12] G. Vahala, P. Pavlo, L. Vahala, N. Martys, "Thermal lattice Boltzmann models for compressible flows", Int. J. Mod. Phys. C, 9, 1247, (1998).

[13] X. He, S. Chen, G. Doolen, "A Novel Thermal Model for the Lattice Boltzmann Method in Incompressible Limit", J. Comp. Phys., 146, 282, (1998).

[14] T. Inamuro, M. Yoshino, F. Ogino, "A non-slip boundary condition for lattice Boltzmann simulations", Phys. Fluids, 7, 2998, (1995).

[15] Q. Zou, X. He, "On pressure and velocity boundary conditions for the lattice Boltzmann BGK model", Phys. Fluids., 9, (1997).

[16] G. H. Tang, W. Q. Tao, Y. L. He, "Simulation of fluid flow and heat transfer in a plane channel using the Lattice Boltzmann method", Int. J. Mod. Phys. B., (2002).

[17] A. D'Orazio, S. Succi, C. Arrighetti, "Lattice Boltzmann simulation of open flows with heat transfer", Phys. Fluids, submitted, (2002).

[18] W. M. Kays, M. E. Crawford, "Convective Heat and Mass Transfer", McGrawHill, Singapore, (1993). 